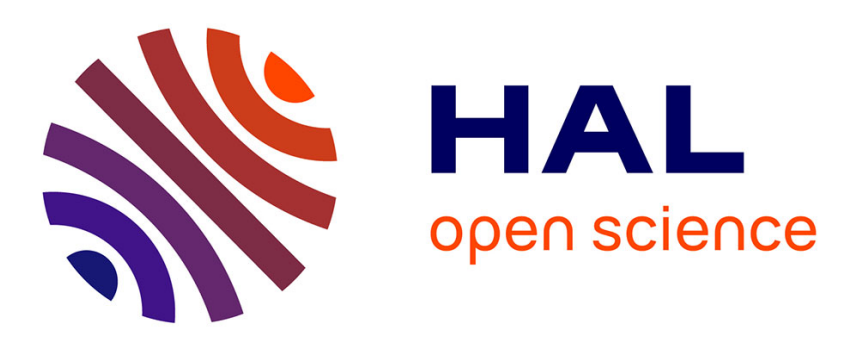

\title{
Physics-conforming constraints-oriented numerical method
}

\author{
Etienne Ahusborde, R. Gruber, M Azaiez, M. L Sawley
}

\section{To cite this version:}

Etienne Ahusborde, R. Gruber, M Azaiez, M. L Sawley. Physics-conforming constraints-oriented numerical method. Physical Review E : Statistical, Nonlinear, and Soft Matter Physics, 2007, 75 (5), pp.056704. 10.1103/PhysRevE.75.056704 . hal-01725473

\section{HAL Id: hal-01725473 \\ https://hal.science/hal-01725473}

Submitted on 7 Mar 2018

HAL is a multi-disciplinary open access archive for the deposit and dissemination of scientific research documents, whether they are published or not. The documents may come from teaching and research institutions in France or abroad, or from public or private research centers.
L'archive ouverte pluridisciplinaire HAL, est destinée au dépôt et à la diffusion de documents scientifiques de niveau recherche, publiés ou non, émanant des établissements d'enseignement et de recherche français ou étrangers, des laboratoires publics ou privés. 


\title{
Physics-conforming constraints-oriented numerical method
}

\author{
E. Ahusborde, ${ }^{1, *}$ R. Gruber, ${ }^{2, \dagger}$ M. Azaiez, ${ }^{1, *}$ and M. L. Sawley ${ }^{2, \S}$ \\ ${ }^{1}$ TREFLE (UMR CNRS 8508), ENSCPB, F-33607 Pessac, France \\ ${ }^{2}$ Laboratory of Computational Engineering, Ecole Polytechnique Fédérale de Lausanne, CH-1015 Lausanne, Switzerland
}

(Received 5 December 2006; revised manuscript received 16 March 2007; published 17 May 2007)

\begin{abstract}
A general high-order finite element method for solving partial differential equations, called the COnstraints Oriented Library (COOL) method, is presented. This $h p$ approach takes into account the underlying nature of the corresponding physical problem, and thus avoids the generation of nonphysical solutions. In the COOL method, all terms in a variational form are represented by the same functional dependence and by the same regularity, thus eliminating regularity constraints imposed by standard numerical methods. External constraints, such as the incompressibility condition appearing in the Maxwell or Navier-Stokes equations, can then be satisfied identically and are eliminated algebraically. This reduces the number of variables, and leads to well-conditioned matrix problems. The consequence is that only physically relevant solutions remain. The COOL method also satisfies automatically internal constraints, such as occur for the grad(div) and curl(curl) operators, and this for any geometry. This approach can be applied to a wide range of physical problems, including fluid flows, electromagnetics, material sciences, ideal linear magnetohydrodynamic stability analysis, and Alfvén wave heating of fusion plasmas. Results obtained by applying the COOL method to the grad(div) and curl(curl) operators, the Stokes problem, and the steady and unsteady Navier-Stokes equations are presented.
\end{abstract}

DOI: 10.1103/PhysRevE.75.056704

\section{INTRODUCTION}

A wide range of physical phenomena can be described by mathematical models based on a set of coupled partial differential equations. A well-known example is the Laplace operator, which arises in many physical problems. This operator is easy to approximate using existing numerical methods. Some operators, however, pose more significant problems, in particular, those that are restricted by physical constraints such as arise from material properties. For example, in most applications, water can be treated as incompressible; mathematically its flow velocity $\boldsymbol{u}$ must satisfy the incompressibility condition $\boldsymbol{\nabla} \cdot \boldsymbol{u}=0$. Current numerical methods can only approximate this condition. As a consequence, the numerical compressibility of water is much greater than its real physical value. For some operators, such as the $\operatorname{grad}(\mathrm{div})$, curl(curl), and magnetohydrodynamic (MHD) operators, the solution is restricted by internal constraints that are an integral part of the solution, and are difficult to satisfy numerically. If these strong internal conditions cannot be satisfied precisely, so-called spectral pollution [1] appears and the numerical approach does not stably converge to the physical solution. The reason is that due to regularity constraints imposed by standard numerical approximation methods, the energy cannot reach the minimum required by the physics. In fact, current numerical methods satisfy the boundary conditions strongly, but satisfy the operator equations and the constraints only weakly.

The COnstraints Oriented Library (COOL) method has been developed based on an entirely different approach. It is

\footnotetext{
*Electronic address: ahusborde@enscpb.fr

${ }^{\dagger}$ Electronic address: ralf.gruber@epfl.ch

*Electronic address: azaiez@enscpb.fr

${ }^{\S}$ Electronic address: mark.sawley@epfl.ch
}

PACS number(s): 02.70.-c, 02.70.Dh, 02.70.Hm

a fundamentally new, mathematically nonconforming numerical method. The idea is to satisfy strongly the constraints and the operator equations, and the boundary conditions only weakly. This implies that this method exactly satisfies external material and internal physical constraints. In addition, each term in the variational form is represented with the same polynomial degree and with the same regularity across element borders. For all cases, the COOL method has the same convergence properties as the established mathematically conforming approximation methods [2] or the spectral method [3]. The COOL approach is an $h p$ method with a general mix of spatial mesh refinement $\left(h \approx \frac{1}{N}\right.$, where $N$ is the number of intervals in each spatial direction) and with a degree $p$ of polynomial basis functions that approximate the solution in each of the $N^{2}$ elements of the two-dimensional geometries considered here. The special case of $p=1$ corresponds to a nonconforming finite element method [1], while $N=1$ corresponds to a new kind of spectral method with the same convergence properties as the standard spectral method.

When applying a numerical method to a physical problem, one should be concerned about nonphysical eigensolutions that can couple with the physical ones, especially for time-evolutionary problems. Due to this concern, it is important to consider the application of numerical methods to eigenvalue problems and demand that only physically relevant eigensolutions are obtained, and to a high degree of precision. If this is assured, the calculated physical solution cannot be affected by the coupling of spurious modes.

The present paper provides a description of the underlying basis of the COOL method for 2D geometries, together with some illustrative examples of its application to different physical problems. The grad(div) problem is presented to show that the spectrum computed using the COOL method is unpolluted even for non-Cartesian meshes. The Stokes prob- 
lem is a good example to demonstrate that the method exactly satisfies $\boldsymbol{\nabla} \cdot \boldsymbol{u}=0$ and that this condition can be eliminated algebraically. Finally, the unsteady and steady NavierStokes equations illustrate how the method is adjusted to run efficiently when iterative sparse matrix solvers are used.

\section{TYPES OF SPATIAL OPERATORS}

In the description of physical phenomena by partial differential equations, we shall distinguish between three different types of spatial operators described in the following sections.

\section{A. Type I: Laplace operator}

The stationary Laplace operator (also called the Poisson equation) can be written as

$$
-\boldsymbol{\nabla} \cdot(k(\mathbf{r}, u) \boldsymbol{\nabla} u)=S(\mathbf{r}, u) \quad \text { for } \mathbf{r} \in \Omega,
$$

restricted by boundary conditions at the border $\partial \Omega$ of the domain $\Omega$. Here, $\boldsymbol{r}$ is the spatial position and $u$ is an unknown scalar function. The coefficient $k(\boldsymbol{r}, u)$ is generally positive. The source term $S(\boldsymbol{r}, u)$ can be complex.

The best known physical phenomenon described by the Laplace operator is the heat equation for which $k=1$ and $S$ $=0$. Other phenomena described by a type I operator are the Schrödinger equation in material science and chemistry, electrostatic potentials, diffusion in chemistry or neutronics, the pressure equation in incompressible fluid flows, the Darcy equation for porous media, solidification processes, and the ideal MHD equilibrium of tokamaks.

All sufficiently regular finite element, finite difference, finite volume, $h p$, or spectral methods can solve the Poisson equation within a discretization error that approaches zero according to well-established convergence laws. Thus, there is no need to develop a new numerical approximation method to solve type I operator problems. The matrices are in general well conditioned, and fast converging iterative matrix solvers can be applied.

\section{B. Type II: Externally constrained operators}

The incompressible Navier-Stokes equations, the Stokes problem, and some special cases of Maxwell's equations are of this type. The numerical specificities of these equations can be represented by the Stokes eigenvalue problem as follows:

$$
\begin{gathered}
-\lambda^{2} \boldsymbol{u}=\Delta \boldsymbol{u}-\nabla p \quad \text { for } \boldsymbol{r} \in \Omega, \\
\boldsymbol{\nabla} \cdot \boldsymbol{u}=0 \quad \text { for } \quad \boldsymbol{r} \in \Omega, \\
\boldsymbol{u}=0 \quad \text { for } \boldsymbol{r} \in \partial \Omega .
\end{gathered}
$$

It is required to compute the eigensolution (eigenvalues and eigenmodes) of the Laplace operator restricted by the incompressibility condition $\boldsymbol{\nabla} \cdot \boldsymbol{u}=0$. The pressure term $\boldsymbol{\nabla} p$ forces the eigenmode to satisfy $\boldsymbol{\nabla} \cdot \boldsymbol{u}=0$.

Mathematically conforming numerical methods cannot exactly reproduce the external incompressibility constraint of

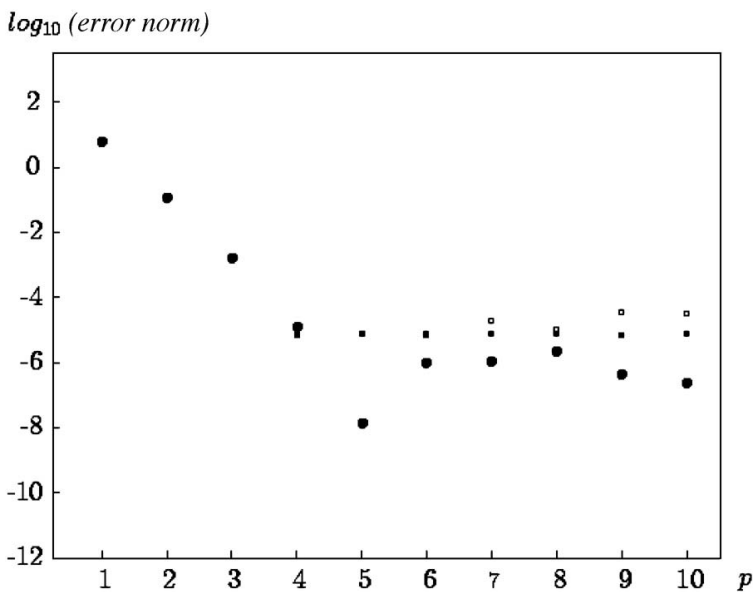

FIG. 1. Convergence plots obtained using the penalty method for the first Stokes mode $\left(\lambda^{2}=13.086173\right)$ as a function of the polynomial order $p$ with fixed $N=4$ for $\alpha=10^{5}$ (small $\bullet$ ), $\alpha=10^{6}$ (large $\bullet$ ), and $\alpha=10^{7}(\circ)$.

the type II class of problems, they only provide an approximation. As a consequence, for example, an incompressible fluid computed using a conforming numerical method becomes slightly compressible. Most of the current numerical approaches used to solve the 2D Stokes problem (2.2) are based either on a penalty method with two variables per mesh point (i.e., the two velocity components) or on the Raviart-Thomas approach with three variables per mesh point (i.e., the two velocities and the pressure) [2]. The problems arising from these approaches are illustrated in Fig. 1 in which the lowest eigenvalue of Eqs. (2.2) is plotted for the penalty method in which the pressure is set to $p=\alpha \boldsymbol{\nabla} \cdot \boldsymbol{u}$, where $\alpha$ is the Lagrange multiplier. One can see that the choice of $\alpha$ leads to slightly different convergence behaviors. For double precision arithmetic, $\alpha=10^{6}$ appears to give the best convergence results. With an increasing polynomial degree to represent the eigenfunction, the eigenvalue converges exponentially as expected for $p \leqslant 5$. Increasing $p$ further does not improve the accuracy of the eigenvalue, with the precision limited to $10^{-6}$. We note here that the limit in precision for the incompressibility condition is of the order of $\sqrt{\alpha}$, thus $\boldsymbol{\nabla} \cdot \boldsymbol{u} \approx 10^{-3}$. Another problem with this popular approach is the appearence of a second class of eigensolutions, the Laplace spectrum multiplied by $\alpha$. This solution, which is not shown in Fig. 1, leads to an ill-conditioned matrix, with a condition number $C \approx 10^{9}$ for $N=p=4$, and consequently, to poor convergence of sparse matrix solvers.

It is possible to satisfy the incompressibility condition precisely by applying the curl operator to the first equation of Eqs. (2.2) and introducing a stream function. The Stokes problem is then described by a double Laplacian [4]. The calculated spectrum is precise, but the computing time is high due to the ill-conditioned resulting matrix. In fact, this precise spectrum is presented in [4] and is used to validate our new COOL approach.

Maxwell's equations are another example of an externally 

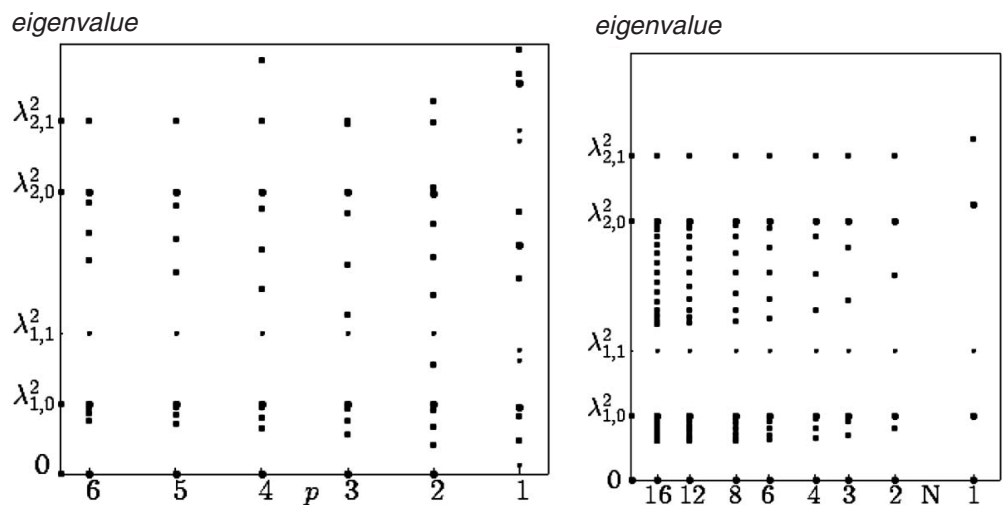

FIG. 2. The dependence of the $\operatorname{grad}($ div) and curl(curl) spectra computed using a standard $h p$ method with $p$ for fixed $N=4$ (left), and with $N$ for fixed $p=4$ (right). The size of the circles distinguish between single, double, and quadruple or higher degenerate eigenvalues. The two lower indexes of the eigenvalue denote the mode numbers in $x$ and $y$ directions.

constrained operator problem. Since it is also internally constrained, we consider this example further in the next section.

\section{Type III: Operators with internal constraints}

Type III operators contain physical constraints embedded in the operator. A typical example is the $\operatorname{grad}(\mathrm{div})$ eigenvalue problem as follows:

$$
\begin{gathered}
-\nabla(\nabla \cdot \boldsymbol{u})=\lambda^{2} \boldsymbol{u} \quad \text { for } \boldsymbol{r} \in \Omega, \\
\boldsymbol{u} \cdot \boldsymbol{n}=0 \quad \text { for } \boldsymbol{r} \in \partial \Omega,
\end{gathered}
$$

representing, for instance, the ideal ocean wave equations [5]. For a 2D geometry, there are two classes of eigensolutions. The incompressible solenoidal modes are infinitely degenerate with $\lambda^{2}=0$ and $\boldsymbol{\nabla} \cdot \boldsymbol{u}=0$. This is the class of modes that give fundamental problems when trying to represent it numerically. The second class of irrotational modes are represented by a discrete spectrum with eigenmodes satisfying $\boldsymbol{\nabla} \times \boldsymbol{u}=0$.

Plasma waves are also described by a type III operator problem [6] as follows:

$$
\begin{gathered}
-\nabla \times \nabla \times \boldsymbol{u}=\lambda^{2} \boldsymbol{u} \quad \text { for } \boldsymbol{r} \in \Omega, \\
\boldsymbol{u} \times \boldsymbol{n}=0 \quad \text { for } \boldsymbol{r} \in \partial \Omega .
\end{gathered}
$$

In $2 \mathrm{D}$, the spectrum of this problem is identical to that described by Eqs. (2.3).

Other type III operators include the ideal linear MHD equations that describe the stability properties of a tokamak [1], and the Maxwell equations [7]. If the vacuum magnetic field $\boldsymbol{B}$ satisfies the initial condition $\boldsymbol{\nabla} \cdot \boldsymbol{B}=0$ at time $t=0$, the Maxwell equations automatically satisfy $\boldsymbol{\nabla} \cdot \boldsymbol{B}=0$ for all $t>0$. If one imposes $\boldsymbol{\nabla} \cdot \boldsymbol{B}=0$ at all $t$, the Maxwell equations are also a type II operator.

The numerical approximation of this type of operator is very difficult. For Cartesian geometries it is possible to choose a staggered grid and different conforming elements for the different vector components $[1,6,8]$. However, if the mesh is not Cartesian, this approach fails due to insufficient regularity of the approximating basis. If a standard approach is then chosen, so-called spectral pollution appears [1]. The class of infinitely degenerate divergence-free eigensolutions expands to a discrete spectrum that changes with $N$ and $p$.
This behavior is illustrated in Fig. 2. The left side of Fig. 2 shows the spectrum for the grad(div) operator calculated using the standard conforming $h p$ elements in a nonCartesian geometry [3]. The number of spatial mesh elements is $N=4$ in both directions, and the polynomial degree is varied between $p=1$ and $p=6$. We note that the solenoidal class of modes should consist of an infinitely degenerate mode with an eigenvalue $\lambda^{2}=0$. For $p=1$ (conforming bilinear finite elements), there is no such eigenvalue, and all the discrete modes of the solenoidal degenerate spectrum are unphysical. For $p>1$, there are $N^{2}(p-1)^{2}$ degenerate eigenvalues with $\lambda^{2}=0$, but also $(N p-1)^{2}-N^{2}(p-1)^{2}-3$ unphysical polluting modes are observed. The irrotational eigenvalues converge exponentially with $p$ towards the analytical solution. Some of these eigenvalues, however, have incorrect multiplicities. The polluting eigensolutions have eigenvalues that lie below those of the irrotational eigensolutions with one wave number equal to zero; thus the first discrete eigenvalue $\lambda_{1,0}^{2}=1$ corresponds to eigensolutions with mode numbers $(0,1)$ and $(1,0)$, and the third eigenvalue $\left(\lambda_{2,0}^{2}=4\right)$ with mode numbers $(0,2)$ and $(2,0)$. With increasing $p$, these unphysical eigenvalues approach the physical one, and the physical and unphysical modes then become increasingly coupled.

The right side of Fig. 2 again shows the polluted spectrum, this time as a function of $N$, fixing $p=4$. One recognizes the presence of continuous spectra below the discrete irrotational modes for which one of the mode numbers in the $x$ or $y$ direction is equal to zero. Two of those spectra can be recognized below the $(0,1)$ and $(1,0)$ and the $(0,2)$ and $(2,0)$ modes. These continuous spectra are unphysical, but are part of the numerical spectrum. In particular, the incorrect degeneracy of the eigenfunction with a mode number 0 in the $x$ or $y$ directions (4 instead of 2) couples the two physical modes of the irrotational spectrum with unphysical modes from the solenoidal class of modes. If such a numerical approach is used for a time-dependent calculation, it is possible that the physical modes couple with unphysical modes, and the resulting solution can be strongly affected.

For a general non-Cartesian geometry, the only conforming approach that is able to compute correctly the spectrum of the $\operatorname{grad}(\mathrm{div})$ operator (2.3) is that based on edge elements, while surface elements represent well the spectrum of the curl(curl) operator [7]. These methods have been formulated for $p=1$. We note, however, that edge elements are not 
suited to solve Eqs. (2.2), since they are not sufficiently regular to approximate the Laplace operator.

We have shown that in certain circumstances, mathematically conforming elements can be adapted to the physics of problems represented by different types of operators. However, in many cases this is not possible, and the approximated solution can then differ significantly from the physical solution. The new COOL method has been developed to compute with high precision the spectra of all type I-III operators, satisfying the constraints exactly, for both Cartesian and non-Cartesian geometries.

\section{NONCONFORMING $h p$ APPROACH}

The nonconforming $h p$ method COOL for solving partial differential equations is presented here by means of the $\operatorname{grad}(\mathrm{div})$ eigenvalue problem (2.3) solved on the square $\Omega=[-1,+1]^{2}$ cut into $N^{2}$ elements. The variational form can be written as Find $\boldsymbol{u}$ sufficiently regular and $\boldsymbol{u} \cdot \boldsymbol{n}=0$ at the boundary $\partial \Omega$ such that

$$
\mathcal{A}(\boldsymbol{u}, \boldsymbol{v}):=\int_{\Omega} \boldsymbol{\nabla} \cdot \boldsymbol{u} \boldsymbol{\nabla} \cdot \boldsymbol{v} d \mathbf{x}=\lambda^{2} \int_{\Omega} \boldsymbol{u} \cdot \boldsymbol{v} d \mathbf{x},
$$

$\forall \boldsymbol{v}$ sufficiently regular. This quadratic, or primal form has the advantage that if $\lambda^{2}=0, \boldsymbol{\nabla} \cdot \boldsymbol{u} \equiv 0$, and no additional tests on the eigenmodes are needed as in [8]. The integral $\mathcal{A}(\boldsymbol{u}, \boldsymbol{v})$ may be expanded as

$$
\mathcal{A}(\boldsymbol{u}, \boldsymbol{v})=\int_{\Omega}\left(\frac{\partial u_{x}}{\partial x}+\frac{\partial u_{y}}{\partial y}\right)\left(\frac{\partial v_{x}}{\partial x}+\frac{\partial v_{y}}{\partial y}\right) d \mathbf{x} .
$$

Normally, one would choose [3]

$$
\begin{aligned}
& u_{x}(x, y) \in \pi_{p}^{1}(x) \otimes \pi_{p}^{1}(y), \\
& u_{y}(x, y) \in \pi_{p}^{1}(x) \otimes \pi_{p}^{1}(y),
\end{aligned}
$$

where $\pi_{p}^{1}$ denotes an approximating polynomial of degree $p$ with a square integrable first order derivative. With such a choice, however, the number of degenerate modes with $\lambda^{2}$ $=0$ does not correspond to the expected number of degrees of freedom. Specifically, for $p=1$ (corresponding to bilinear finite elements) there is no such mode [see Fig. 2(left)] in addition, unphysical eigensolutions are calculated as previously described.

To obtain the couple $\left(\lambda^{2}=0, \boldsymbol{\nabla} \cdot \boldsymbol{u}=0\right)$ correctly, we modify the variational formulation. Instead of Eqs. (3.1) and (3.2) we consider

$$
\begin{gathered}
\int_{\Omega}\left(\frac{\partial u_{x}^{(1)}}{\partial x}+\frac{\partial u_{y}^{(2)}}{\partial y}\right)\left(\frac{\partial v_{x}^{(1)}}{\partial x}+\frac{\partial v_{y}^{(2)}}{\partial y}\right) d \mathbf{x} \\
=\lambda^{2} \int_{\Omega}\left(u_{x}^{(0)} v_{x}^{(0)}+u_{y}^{(0)} v_{y}^{(0)}\right) d \mathbf{x} .
\end{gathered}
$$

The number of unknowns has been extended from two $\left(u_{x}, u_{y}\right)$ to six $\left(u_{x}^{(k)}, u_{y}^{(k)}, k=0,1,2\right)$ for the most general case when all the partial derivatives appear in the operator. The goal is now to approximate the new variables such that all terms in the operator have the same functional dependencies and the same regularities as follows:

$$
\begin{gathered}
u_{r}^{(0)}(x, y) \in \pi_{p-1}^{0}(x) \otimes \pi_{p-1}^{0}(y), \\
u_{r}^{(1)}(x, y) \in \pi_{p}^{1}(x) \otimes \pi_{p-1}^{0}(y), \\
u_{r}^{(2)}(x, y) \in \pi_{p-1}^{0}(x) \otimes \pi_{p}^{1}(y) .
\end{gathered}
$$

The upper index 0 of $\pi$ denotes a square integrable property, and $r$ denotes $x$ or $y$. With such a choice

$$
\boldsymbol{\nabla} \cdot \boldsymbol{u} \in \pi_{p-1}^{0}(x) \otimes \pi_{p-1}^{0}(y),
$$

and $\boldsymbol{\nabla} \cdot \boldsymbol{u}$ can vanish identically when required. These new variables are related to the initial variables by momentum conditions as follows:

$$
\int_{\Omega}\left(u_{r}-u_{r}^{(k)}\right) \mu d \mathbf{x}=0 \quad \forall k \in\{0,1,2\}, \quad r=x \text { or } y,
$$

where $\mu=x^{\alpha} y^{\beta}$, with $\alpha$ and $\beta=0,1, \ldots, p-1$.

Let us introduce two canonical polynomial basis functions

$$
\begin{aligned}
h_{j}(r) & =-\frac{1}{p(p+1)} \frac{1}{L_{p}\left(\xi_{j}\right)} \frac{\left(1-r^{2}\right) L_{p}^{\prime}(r)}{\left(r-\xi_{j}\right)}, \\
-1 & \leqslant r \leqslant+1, \quad 0 \leqslant j \leqslant p,
\end{aligned}
$$

$$
g_{j}(r)=h_{j}(r)-\frac{L_{p}(r)}{(p+1) L_{p}\left(\xi_{j}\right)}, \quad-1 \leqslant r \leqslant+1, \quad 0 \leqslant j \leqslant p,
$$

where $L_{p}$ is the Legendre polynomial of degree $p, L_{p}^{\prime}$ its derivative with respect to $r$, and $\xi_{j}, j=0,1, \ldots, p$, are the zeros of $\left(1-r^{2}\right) L_{p}^{\prime}(r)=0$. Since $L_{p}(r)$ is zero at the Gauss points $\zeta_{i}, i=1, \ldots, p$, we have $h_{j}\left(\zeta_{i}\right)=g_{j}\left(\zeta_{i}\right)$. The function $h_{j}(r)$ is a polynomial of degree $p$, continuous across element borders. It is used to represent derivatives that are polynomials of degree $p-1$, discontinuous across element borders. The function $g_{j}(r)$ is a polynomial of degree $p-1$, discontinuous across element borders, and is used to represent variations in directions without derivatives. As a consequence, each term in the variational form and in the $\boldsymbol{\nabla} \cdot \boldsymbol{u}$ term (3.6) are polynomials of degree $p-1$ in $x$ and $y$, discontinuous across element borders.

We can then approximate

$$
\begin{aligned}
& u_{r}^{(0)}(x, y)=\sum_{e=1}^{N} \sum_{f=1}^{N} \sum_{i=\delta_{e 1}}^{p} \sum_{j=\delta_{f 1}}^{p} \bar{u}_{i j}^{r e f} g_{i}\left(x_{e f}\right) g_{j}\left(y_{e f}\right), \\
& u_{r}^{(1)}(x, y)=\sum_{e=1}^{N} \sum_{f=1}^{N} \sum_{i=\delta_{e 1}}^{p} \sum_{j=\delta_{f 1}}^{p} \bar{u}_{i j}^{r e f} h_{i}\left(x_{e f}\right) g_{j}\left(y_{e f}\right),
\end{aligned}
$$




\begin{tabular}{|c|c|c|c|c|c|c|c|c|c|c|c|}
\hline $\mathrm{O}$ & 0 & & 0 & 0 & 0 & $x$ & $\bar{x}$ & & $\bar{x} \times$ & $\bar{x}$ & $\begin{array}{ll}x & x\end{array}$ \\
\hline & & & & & & $x$ & $x$ & $x$ & $\times \times$ & $x$ & $x$ \\
\hline 0 & 0 & O & 10 & 0 & 0 & & & & & & \\
\hline & & & & & & $x$ & $\times$ & $\times$ & $\times x$ & $x$ & $\times \quad \times$ \\
\hline$\square$ & $\square$ & 0 & $\square$ & $\square$ & 0 & $x$ & $x$ & $x$ & $\times x$ & $\underline{x}$ & \\
\hline$\square$ & 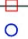 & 0 & 0 & 0 & 0 & $x$ & $\bar{x}$ & $x$ & \begin{tabular}{l|l}
$x$ & $x$ \\
\end{tabular} & $\bar{x}$ & $\begin{array}{ll}x & \times\end{array}$ \\
\hline & & & & & & $x$ & $x$ & $x$ & $\times \mid x$ & $x$ & $\times$ \\
\hline 0 & 0 & $\mathrm{O}$ & 80 & 0 & 0 & & & & & & \\
\hline & & & & & & $x$ & $x$ & $x$ & $x \times$ & $x$ & $x$ \\
\hline 口 & $\square$ & & $p \square$ & $\square$ & 0 & $\times$ & $x$ & $x$ & $x \mid x$ & $x$ & $x$ \\
\hline
\end{tabular}

FIG. 3. (Color online) Left: Position of the variables $\overline{\boldsymbol{u}}$ at the GLL points for $N=2, p=4$. The circles (blue) denote the eliminated components $\boldsymbol{u}_{2}$ and the squares (red) the remaining variables $\boldsymbol{u}_{1}$. Note that each circle or square contains two variables corresponding to the local $\left(\bar{u}_{x}, \bar{u}_{y}\right)$ components. There are no variables needed on the boundary, since $\overline{\boldsymbol{u}}=0$ there. Right: The Gauss points (green crosses) for the integration and elimination of the $\boldsymbol{\nabla} \cdot \boldsymbol{u} \equiv 0$ conditions.

$$
u_{r}^{(2)}(x, y)=\sum_{e=1}^{N} \sum_{f=1}^{N} \sum_{i=\delta_{e 1}}^{p} \sum_{j=\delta_{f 1}}^{p} \bar{u}_{i j}^{r e f} g_{i}\left(x_{e f}\right) h_{j}\left(y_{e f}\right) .
$$

Here, $\delta_{e 1}$ and $\delta_{f 1}$ are the Kronecker symbols and the index $r$ denotes $x$ or $y$. There are $2(N p)^{2}$ variables for $\overline{\boldsymbol{u}}$, whereas there are $2(N p+1)^{2}$ variables for $\boldsymbol{u}$ defined in Eq. (3.3). The reason is that the function $g(r)$ is a polynomial of degree $p-1$, implying that one has to eliminate variables if there is no boundary condition at the lower or upper border in $x$ or at the left or right border in $y$. The lower border variables $\bar{u}_{i 0}^{y e 1}$ or the left side variables $\bar{u}_{0 j}^{x 1 f}$ have then to be eliminated to obtain regular matrices. All the quantities in Eqs. (3.10)-(3.12) are identical at the Gauss points. The dependent variables $\overline{\boldsymbol{u}}$ have two vector components. They are defined at the Gauss-Lobatto-Legendre (GLL) points [see Fig. 3 (left)]. If one has to impose regularity conditions, these components have no physical meaning, only the sum defined before. Using these expressions one can easily verify that the moment equations (3.7) are satisfied. In addition, all the terms in the variational form (3.4) are polynomials of degree $p-1$ with a jump across the element borders.

\section{SATISFYING EXTERNAL CONSTRAINTS USING THE COOL METHOD}

\section{A. Elimination of $\nabla \cdot u=0$}

Constraints such as $\boldsymbol{\nabla} \cdot \boldsymbol{u}=0$ are often imposed through a penalty function [2] or by introducing a stream function [4]. Another way to impose such a constraint is to find a precisely divergence-free basis as the eigenmodes that form the kernel of the $\operatorname{grad}($ div) operator. This leads to a perfect Stokes spectrum. The drawback of this method is that the matrices of the eigenvalue problem are full, making it inapplicable to real-world 3D problems. We shall present an alternative method in which the matrices remain sparse.

The 2D Stokes eigenvalue problem (2.2) in variational form can be written as Find $\boldsymbol{u}$ sufficiently regular satisfying $\boldsymbol{\nabla} \cdot \boldsymbol{u}=0$, and $\boldsymbol{u}=0$ on $\partial \Omega$ and the real quantity $\lambda$ such that

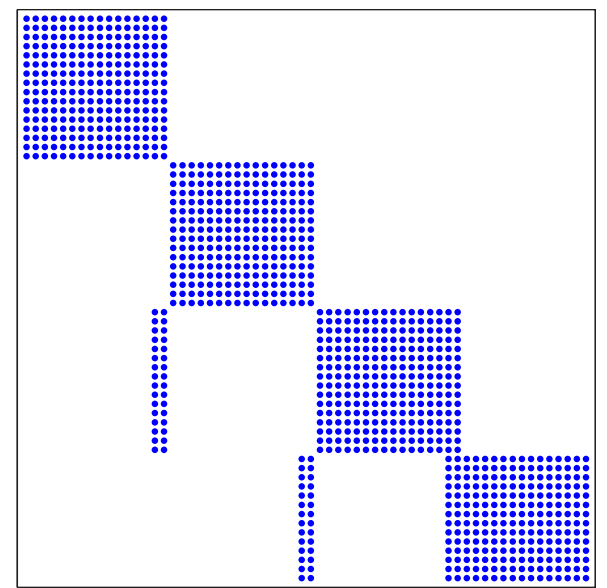

FIG. 4. (Color online) The sparsity pattern of $D_{2}$ and its decomposition matrices $L$ and $U$ for $N=2$ and $p=4$. The rank of the entire matrices is $(N p)^{2}-2=62$. All matrix blocks have a rank of $p^{2}=16$, except the last block that has a rank of $p^{2}-2=14$. The white parts in the matrix are filled with zeros.

$$
\mathcal{S}(\boldsymbol{u}, \boldsymbol{v})=\int_{\Omega} \boldsymbol{\nabla} \boldsymbol{u} \cdot \nabla \boldsymbol{v} d \mathbf{x}=\lambda^{2} \int_{\Omega} \boldsymbol{u} \cdot \boldsymbol{v} d \mathbf{x}, \quad \forall \boldsymbol{v}
$$

This problem has $N_{v a r}=2(N p-1)^{2}$ variables. It is solved in two steps applying the new constraint-oriented, nonconforming $h p$ method COOL. First, $\boldsymbol{\nabla} \cdot \boldsymbol{u}=0$ is imposed at the $(N p)^{2}$ Gauss points [see Fig. 3 (right)], leading to one algebraic equation per Gauss point. This equation includes all variables in the element. Due to the boundary conditions, in the present case $\boldsymbol{u}=0$, two of these linear equations are linearly dependent.

Thus, one can eliminate $N_{2}=(N p)^{2}-2$ variables that are combined in $\boldsymbol{u}_{2}$ [see Fig. 3 (left)]. The remaining $N_{1}=(N p-2)^{2}$ variables are included in $\boldsymbol{u}_{1}$. Note that $N_{v a r}$ $=N_{1}+N_{2}$. The $\boldsymbol{\nabla} \cdot \boldsymbol{u}=0$ equations expressed at the $N_{2}$ Gauss points can then be written in matrix form as follows:

$$
D_{1} \boldsymbol{u}_{1}+D_{2} \boldsymbol{u}_{2}=0
$$

Here, $D_{1}$ is a matrix with $N_{2}$ rows and $N_{1}$ columns and $D_{2}$ has $N_{2}$ rows and $N_{2}$ columns. Then,

$$
\boldsymbol{u}_{2}=-D_{2}^{-1} D_{1} \boldsymbol{u}_{1}
$$

leading to an algebraic matrix condition between the initial $\bar{u}$ variables and the new remaining variables $\boldsymbol{u}_{1}$ as follows:

$$
\overline{\boldsymbol{u}}=M \boldsymbol{u}_{1}
$$

The matrix

$$
M=\left(\begin{array}{c}
I \\
-D_{2}^{-1} D_{1}
\end{array}\right)
$$

has $N_{v a r}$ rows and $N_{1}$ columns. Instead of inverting matrix $D_{2}$, it is decomposed into 
TABLE I. Number of iterations required for the Laplacian matrix $A$ (left), the symmetric Stokes matrix $M^{T} A M$ (center), and the new Stokes matrix $N^{T} A M$ (right).

\begin{tabular}{lccc}
\hline \hline$p=N$ & Laplacian & Stokes & Stokes \\
$M^{T} A M$ & $N^{T} A M$ \\
\hline 3 & $A$ & 59 & 29 \\
4 & 16 & 289 & 54 \\
5 & 34 & 925 & 118 \\
6 & 62 & 2613 & 189 \\
\hline \hline
\end{tabular}

$$
D_{2}=L U,
$$

keeping unchanged the sparsity pattern of $D_{2}$ shown in Fig. 4.

The initial eigenvalue problem

$$
A \boldsymbol{u}=\lambda^{2} B \boldsymbol{u},
$$

derived from Eq. (4.1), then becomes

$$
M^{T} A M u_{1}=\lambda^{2} M^{T} B M u_{1} .
$$

The multiplication by $M^{T}$ from the left guarantees that the generalized eigenvalue problem remains symmetric.

\section{B. Condition numbers of the matrix problems}

The condition number of the matrix $\left(M^{T} B M\right)^{-1} M^{T} A M$ is $C=900$ for $N=4$ and $p=4$. In the case of the generalized eigenvalue problem of a Laplace operator, the matrix $B^{-1} A$ has a condition number $C=4220$ for $N=4$ and $p=4$. This means that the Stokes eigenvalue problem is better conditioned than the Laplace eigenvalue problem.

When applying this method to time-dependent problems, the condition number of the Lapacian matrix $A$ is important. This condition number is $C=130$, sufficiently well conditioned to use a conjugate gradient (CG) method, even without preconditioning. On the other hand, the condition number of the matrix $M^{T} A M$ is $C=357323$. This is almost the square of the condition number for the Stokes eigenvalue problem. We note that the matrix multiplication by $M^{T}$ is not a sparse matrix operation.

To overcome these problems, it is proposed to multiply the initial matrix problem (4.7) from the left hand side with a general matrix $N^{T}$ instead of multiplying by $M^{T}$. For instance, for the unsteady Navier-Stokes problem presented later, the initial problem

$$
A M \boldsymbol{u}_{1}=f
$$

becomes

$$
N^{T} A M \boldsymbol{u}_{1}=N^{T} \boldsymbol{f} .
$$

The simplest choice for $N^{T}$ is

$$
N=\left(\begin{array}{l}
I \\
0
\end{array}\right) \text {. }
$$

With such a transformation matrix, the condition number of $N^{T} A M$ for $N=4$ and $p=4$ becomes $C=3008$. This matrix is

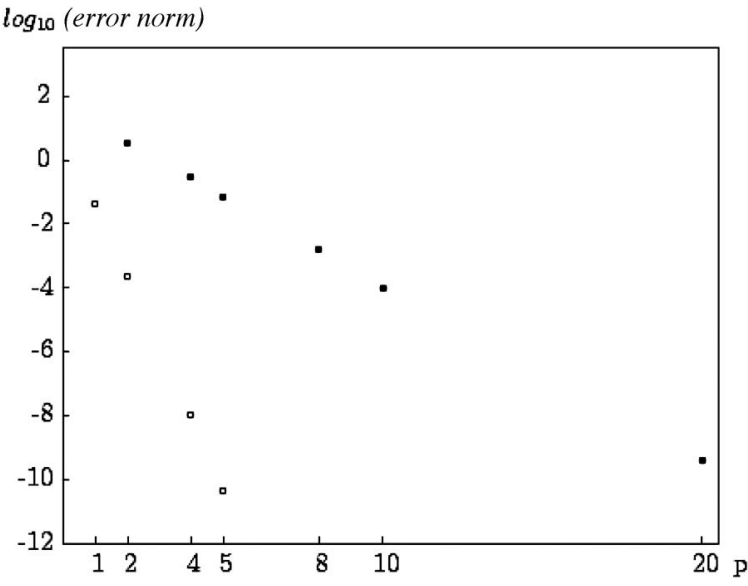

FIG. 5. Convergence plots for the first $\left(\lambda_{1}^{2}=13.0861727921\right.$, open circles) and the $73 \mathrm{rd}\left(\lambda_{73}^{2}=301.8406425660\right.$, closed circles) Stokes modes obtained using the COOL method with a constant value of $N p=40$. In both cases, exponential convergence is observed until machine precision is achieved.

no longer symmetric and positive definite, but unsymmetric and thus requires the use of a biconjugate gradient (BiCG) iterative matrix solver demanding twice as many operations as the $\mathrm{CG}$ solver. For this test case, the number of necessary $\mathrm{CG}$ and $\mathrm{BiCG}$ iteration steps without preconditioning is shown in Table I. It is seen that a time-dependent Stokes problem requires twice as many iteration steps as a stationary Laplacian, whereas many more iteration steps are needed for the symmetric Stokes problem.

The new matrix problem can be solved iteratively with sparse matrix operations. The $N^{T} A$ multiplication is trivial. It implies that all the rows of $A$ below row number $N_{1}$ can be set to zero, and the number of operations therefore diminishes. To accelerate further the iteration process, the identity matrix in $N^{T}$ could be replaced by a preconditioner.

\section{Application to the Stokes eigenvalue problem}

The Stokes spectrum is discrete in nature, similar to that of the Laplace operator. There are no singular eigenvalues, thus the spectrum cannot be polluted. To demonstrate the efficiency of the COOL method to solve externally constrained problems, we apply it to this well-known spectrum. The eigenvalues and eigenmodes are found with high precision, and no modes other than the Stokes modes are found. The total number of mesh points in both directions is fixed at $N p=40$. Figure 5 shows the convergence behavior of the first $\left(\lambda_{1}^{2}=13.0861727921\right)$ and the 73rd $\left(\lambda_{73}^{2}=301.8406425660\right)$ eigenvalues to machine precision with ten decimal places. The calculation of the eigenvalues is observed to converge exponentially. We note that for $p=20$ all ten decimal places of the calculated eigenvalues correspond to those reported in [4].

It has been shown theoretically that the eigenmodes have a global structure with an infinite series of Moffatt corner vortices of increasingly smaller amplitudes [9]. For the 13th eigenmode with $\lambda_{13}^{2}=69.769769316$, the $u_{x}$ component of the eigensolution is shown on the right side of Fig. 6 using 


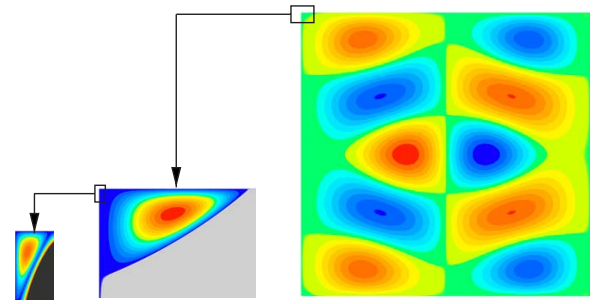

FIG. 6. (Color online) The $u_{x}$ component of the 13th eigenmode computed using the COOL method. The first and second Moffatt corner vortices are shown in the insets at the center and left, respectively. The sign of the eigenmode structure changes from subdomain (red/yellow) to subdomain (blue/green).

one element and $p=32$. The amplitude of this global structure is 0.852 . The first Moffatt vortex in the left upper corner of the geometry has an amplitude of $10^{-3}$, while the second Moffatt corner vortex has an amplitude of $2 \times 10^{-6}$, this being close to machine precision. The detection of the third Moffatt corner vortex would necessitate quadruple precision arithmetic.

\section{COOL METHOD APPLIED TO INTERNAL CONSTRAINTS}

The eigensolution of operators with internal constraints include infinitely degenerate finite value eigenvalues. These can exhibit an infinite degeneracy as for the $\operatorname{grad}(\mathrm{div})$ operator (2.3), an accumulation point, or a continuous spectrum as for ideal linear MHD [1]. Accurate determination of these degeneracies can only be achieved if the numerical method is able to satisfy the constraints identically. Otherwise, the approximated solution will not stably converge towards the physical one, and spectral pollution occurs as shown in Fig. 2.

In the COOL method, all terms of the operator have the same functional dependence and the same regularities across the element borders. The sum of terms can then identically vanish. As a consequence, the COOL method always auto-

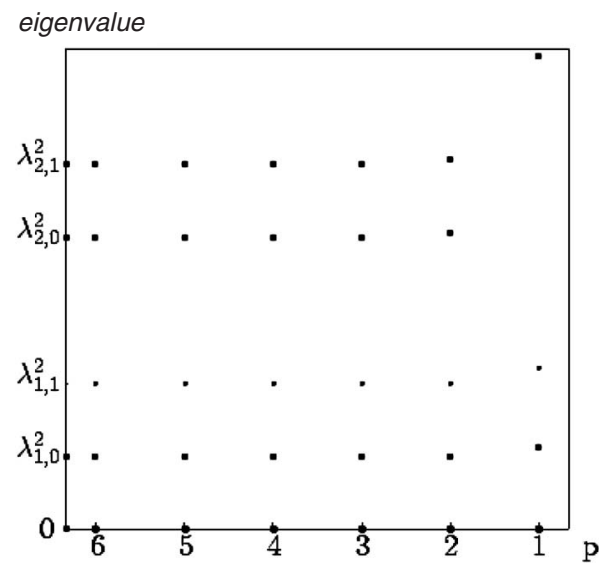

FIG. 7. The dependence on $p$ of the $\operatorname{grad}($ div) and curl(curl) spectra computed using the COOL method for fixed $N=4$. The two lower indexes of the eigenvalue denote the mode numbers in the $x$ and $y$ directions. matically satisfies internal constraints even though one cannot always analytically recognize them. The ideal linear MHD problem in a complex geometry is such an example where the COOL method reveals its full power.

\section{A. Grad(div) and curl(curl) eigenvalue problems}

Figure 7 shows the spectra of the grad(div) and curl(curl) operators obtained by applying the COOL method. The number of intervals is fixed at $N=4$, and $p$ varies between 1 and 6 as in Fig. 2 (left). All modes calculated are physical. The eigenvalue $\lambda^{2}=0$ is obtained with the expected $(N p-1)^{2}$-fold degeneracy. For $p=4$, eight decimal places are correct for the lowest eigenvalues. The convergence in $p$ is exponential. This figure is to be compared with Fig. 2 (left). One clearly recognizes that the original unphysical spectrum has been replaced by a physical spectrum. This is the case for both Cartesian and non-Cartesian geometries.

\section{B. Ideal linear MHD equations}

The ideal linear MHD operator, which describes the stability and Alfvén wave heating properties of fusion plasmas in tokamaks or stellerators, includes two continuous spectra with complex internal constraints. To improve precision, magnetic flux surfaces have to be well represented. This implies that any mathematically conforming method is definitely not suited for this operator. The COOL method for $p=1$ is not only able to predict correctly the stability behaviors of tokamaks [10] but also Alfvén wave heating mechanisms [11] (resonance absorption of a global mode by the continuum) used to heat fusion reactor experiments.

\section{APPLICATIONS TO UNSTEADY AND STEADY NAVIER-STOKES PROBLEMS}

To examine the COOL method applied to the unsteady Navier-Stokes problem, we consider the equations set on $\Omega \times\left[0, t^{*}\right]$ as follows:

$$
\begin{gathered}
\frac{\partial \boldsymbol{u}}{\partial t}+(\boldsymbol{u} \cdot \nabla) \boldsymbol{u}-\nu \Delta \boldsymbol{u}=\boldsymbol{f} \quad \text { in } \Omega \times\left[0, t^{*}\right], \\
\operatorname{div} \boldsymbol{u}=0 \quad \text { in } \Omega \times\left[0, t^{*}\right], \\
\boldsymbol{u}=0 \quad \text { on } \partial \Omega \times\left[0, t^{*}\right], \\
\boldsymbol{u}(\cdot, t=0)=0 \quad \text { in } \Omega .
\end{gathered}
$$

The numerical method begins with the treatment of the nonlinear term $(\boldsymbol{u} \cdot \boldsymbol{\nabla}) \boldsymbol{u}$ involved in the material derivative of the velocity, $\frac{d u}{d t}\left[=\frac{\partial u}{\partial t}+(\boldsymbol{u} \cdot \nabla) \boldsymbol{u}\right]$. The scheme adopted here is the classical explicit second-order Adams-Bashforth algorithm. In order to specify our scheme, we divide the time interval of integration $\left[0, t^{*}\right]$ into $m^{*}$ subdivisions of length $\Delta t=\frac{t^{*}}{m^{*}}$, and define $t^{m}=m \Delta t$, for any $m, 0 \leqslant m \leqslant m^{*}$. We shall compute a sequence $\left(\boldsymbol{u}^{m}\right)_{0 \leqslant m \leqslant m}$ in a recurrent way that approximates in some sense $\left(\boldsymbol{u}\left(\cdot, t^{m}\right)\right)_{0 \leqslant m \leqslant m^{*}}$. Assuming $\left(\boldsymbol{u}^{k}\right)_{0 \leqslant k \leqslant m}$ to be known, we then determine the velocity $\boldsymbol{u}^{m+1}$ by solving 


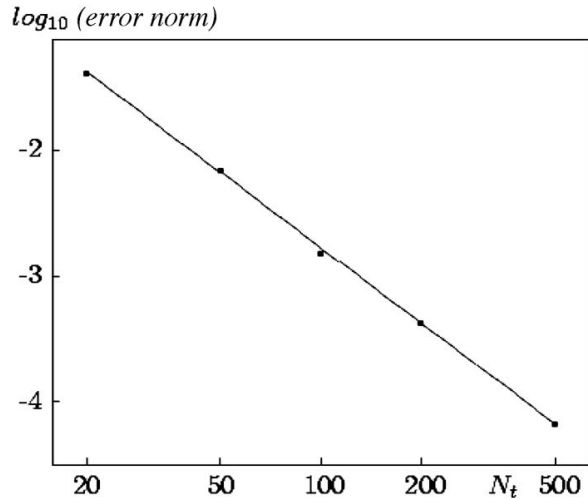

FIG. 8. Convergence plot obtained for the unsteady NavierStokes solution using the COOL method. The straight line corresponds to quadratic convergence with the number of time steps $N_{t}$.

$$
\begin{gathered}
\frac{3 \boldsymbol{u}^{m+1}-4 \boldsymbol{u}^{m}+\boldsymbol{u}^{m-1}}{2 \Delta t}-\nu \Delta \boldsymbol{u}^{m+1} \\
=\boldsymbol{f}^{m+1}-\operatorname{NLN}\left(\boldsymbol{u}^{m}, \boldsymbol{u}^{m-1}\right) \quad \text { in } \Omega, \\
\operatorname{div} \boldsymbol{u}^{m+1}=0 \quad \text { in } \Omega, \\
\boldsymbol{u}^{m+1}=0 \quad \text { on } \partial \Omega,
\end{gathered}
$$

where $N L N\left(\boldsymbol{u}^{m}, \boldsymbol{u}^{m-1}\right)=2((\boldsymbol{u} \cdot \nabla) \boldsymbol{u})^{m}-((\boldsymbol{u} \cdot \nabla) \boldsymbol{u})^{m-1}$.

To check the dependence of the accuracy with respect to the temporal approximation, we solve the unsteady NavierStokes equations with $\nu=1$ having the following analytical solution:

$$
\boldsymbol{u}=\left(\begin{array}{c}
\sin (y) \cos (5 t) \\
\sin (x) \cos (5 t)
\end{array}\right) \text {. }
$$

Values of $p=6$ and $N=2$ have been chosen for the computation. In Fig. 8 is presented, on a logarithmic scale, the error with respect to the discrete maximum norm at time $t=1$. The expected second-order decrease of the error with the number of time steps $N_{t}$ is observed.

The second example presented here was first studied by Kovasznay in 1948 [12], and represents laminar flow behind a 2D grid. We consider the resolution of the steady

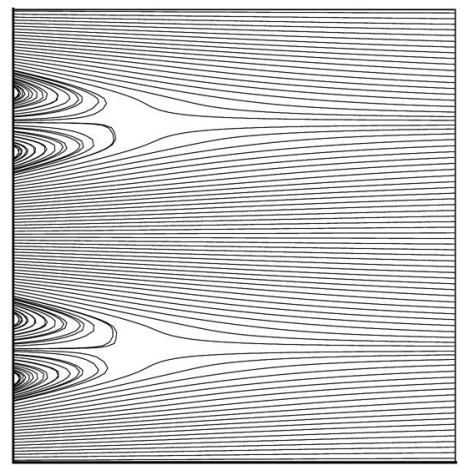

FIG. 9. Streamlines computed for the Kovasznay flow problem on the rectangular domain $\Omega=(-0.5,1) \times(-0.5,1.5)$. These results are in complete agreement with Eq. (6.9).
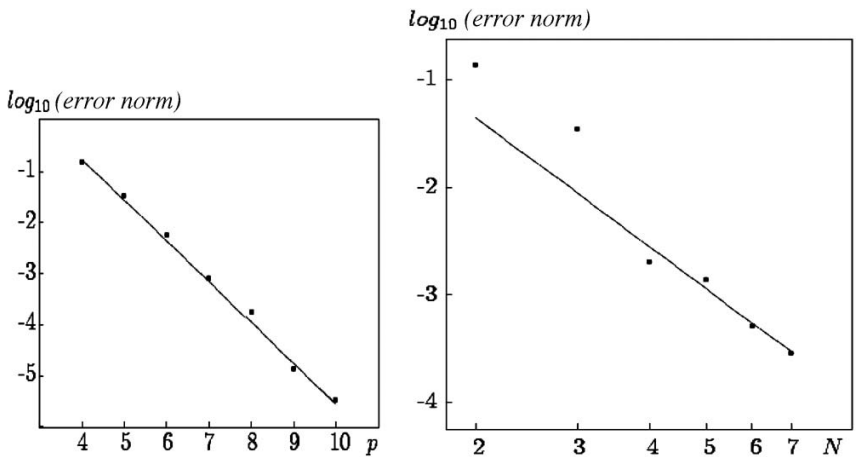

FIG. 10. Convergence plot for the Kovasznay solution with respect to $p$ (left) and $N$ (right).

Navier-Stokes equations on the rectangular domain $\Omega=(-0.5,1) \times(-0.5,1.5)$, with the exact solution given by

$$
\boldsymbol{u}=\left(\begin{array}{c}
1-e^{\lambda x} \cos (2 \pi y) \\
\frac{\lambda}{2 \pi} e^{\lambda x} \sin (2 \pi y)
\end{array}\right),
$$

where $\lambda=\frac{\mathrm{Re}}{2}-\left(\frac{\mathrm{Re}^{2}}{4}+4 \pi^{2}\right)^{1 / 2}$, and Re is the Reynolds number which has a value of 40. The solution, shown in Fig. 9, can be obtained as the limit (when $t \rightarrow+\infty$ ) of the unsteady Navier-Stokes equations, starting for instance from a zero initial condition. At each time step (for $\Delta t=0.01$ ), the semidiscrete generalized Stokes problem (6.5)-(6.7) is solved using the COOL method. The time marching is terminated when the steady regime is established, that is, when the difference between the velocities computed at two consecutive time steps is less than a predefined tolerance, $\max \left(\left|\boldsymbol{u}_{i j}^{m+1}-\boldsymbol{u}_{i j}^{m}\right|\right)<1 \times 10^{-8}$.

Figure 10 (left) presents, in a semilogarithmic plot, the dependence of the maximum norm error with respect to $p$, fixing $N=2$. Due to the infinitely smooth nature of $\boldsymbol{u}$, the expected exponential decrease in the error is observed. In Fig. 10 (right) is presented, in a logarithmic plot, the dependence of the maximum norm error with respect to $N$, fixing $p=4$. The expected algebraic $\mathcal{O}\left(N^{-p}\right)$ decrease is observed.

\section{CONCLUSIONS}

A new, general numerical method for solving partial differential equations has been presented. This method differs from existing methods in that it conforms exactly to the physical contraints imposed. This results in a number of advantages, which have been illustrated by the application of the method to different physical problems.

\section{A. COOL method for externally constrained problems}

The strengths of the COOL method applied to problems with external constraints are

(1) The number of dependent variables in the 2D Stokes problem reduces from three $\left(u_{x}, u_{y}, p\right)$ to one $\left(u_{1}\right)$ component, and the matrix problem is thus much smaller.

(2) Only Stokes modes are obtained. 
(3) The $\boldsymbol{\nabla} \cdot \boldsymbol{u}=0$ is satisfied exactly, and thus the material constraints are exactly reproduced.

(4) The matrix $M$ has a sparsity pattern similar to $A$, and thus computations can be performed in a fully parallel manner.

(5) The condition number of the $\left(M^{T} B M\right)^{-1} M^{T} A M$ matrix is smaller than for the Laplace problem $B^{-1} A$.

(6) The eigensolution of the 2D Stokes spectrum converges exponentially in $p$ as for conforming $h p$ methods [3].

(7) The COOL method is valid for Cartesian and nonCartesian geometries.

\section{B. COOL method for internally constrained problems}

The strengths of the COOL method applied to problems with internal constraints are

(1) There is no spectral pollution [1].

(2) The COOL method automatically adapts to the underlying physics and reproduces it correctly.

(3) The eigensolution of the 2D spectra converges exponentially in $p$ as for conforming $h p$ methods [3].

(4) The COOL method is valid for Cartesian and nonCartesian geometries.

(5) The COOL method can be applied to the primal and to the dual variational form [8] without leading to spectral pollution.

\section{COOL method for time-dependent problems}

The strengths of the COOL method applied to timedependent constraint problems are

(1) Constraints are exactly satisfied.

(2) The number of variables is reduced to the number that the physics imposes.

(3) Using $N^{T}$ as the transformation matrix, the overall matrix problem reduces in size and enables sparse matrix operations.

(4) The condition number of $N^{T} A M$ is of the same order of magnitude as for the Laplace operator.

(5) Only physically relevant solutions are obtained, thus coupling to unphysical modes is impossible.

Given the generality of the COOL method and the numerous advantages it provides for the resolution of externally and internally constrained problems of both stationary and unsteady nature, it is foreseen that this method will find application to partial differential equations arising in a wide range of physical problems.

\section{ACKNOWLEDGMENTS}

Special thanks to Michel Deville for his very constructive remarks on the paper and to Ernest Mund for his help in the formulation of the COOL method.
[1] R. Gruber and J. Rappaz, Finite Element Methods in Linear Ideal MHD, Springer Series in Computational Physics (Springer-Verlag, Berlin, 1985).

[2] V. Girault and P. Raviart, Finite Element Methods for NavierStokes Equations, Springer Series in Computational Mathematics (Springer-Verlag, Berlin, 1986).

[3] M. O. Deville, P. F. Fischer, and E. H. Mund, High-Order Methods for Incompressible Fluid Flow (Cambridge University Press, Cambridge, 2002).

[4] E. Leriche and G. Labrosse, J. Comput. Phys. 200, 489 (2004).

[5] J. Descloux, M. Luskin, and J. Rappaz, Math. Comput. 36, 137 (1981).

[6] M.-C. Festeau-Barrioz and E. S. Weibel, Comput. Phys. Com- mun. 27, 11 (1982).

[7] A. Bossavit, Computational Electromagnetism (Academic Press, New York, 1998).

[8] D. Boffi, F. Brezzi, and L. Gastaldi, Math. Comput. 69, 121 (1999).

[9] H. K. Moffatt, J. Fluid Mech. 18, 1 (1964).

[10] F. Troyon, R. Gruber, H. Saurenmann, S. Semenzato, and S. Succi, Plasma Phys. Controlled Fusion 26, 209 (1984).

[11] K. Appert, R. Gruber, F. Troyon, and J. Vaclavik, Plasma Phys. 24, 903 (1982).

[12] L. I. G. Kovasznay, Proc. Cambridge Philos. Soc. 44, 58 (1948). 